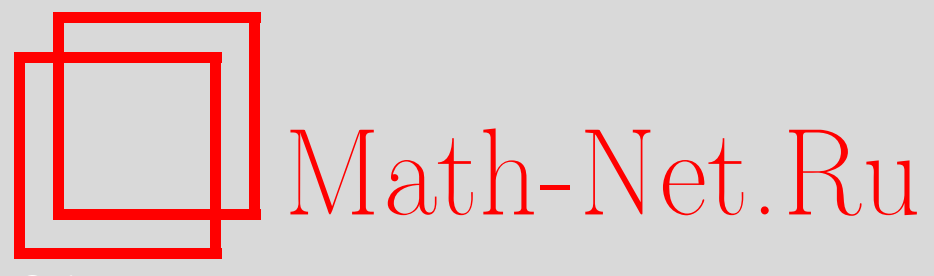

Н. Р. Садыков, Поляризационные эффекты, обусловленные взаимным влиянием параметров траектории и поляризации, ТМФ, 2006, том 149, номер 1, 65-79

DOI: https://doi.org/10.4213/tmf3832

Использование Общероссийского математического портала Math-Net.Ru подразумевает, что вы прочитали и согласны с пользовательским соглашением http: //www . mathnet.ru/rus/agreement

Параметры загрузки:

IP: 3.95 .254 .165

26 апреля 2023 г., 17:06:33

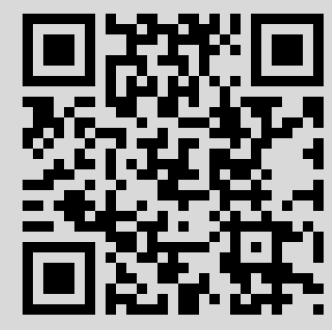




\title{
ПОЛЯРИЗАЦИОННЫЕ ЭФФЕКТЫ, ОБУСЛОВЛЕННЫЕ ВЗАИМНЫМ ВЛИЯНИЕМ ПАРАМЕТРОВ ТРАЕКТОРИИ И ПОЛЯРИЗАЦИИ
}

\begin{abstract}
В приближении геометрической оптики проведен сравнительный анализ поляризационных эффектов, являющихся следствием влияния поляризации на траекторию пучка лучей. Показано, что уравнения траектории пучка лучей, описывающие оптический эффект Магнуса, которые получены на основе канонических уравнений Гамильтона, принципа Ферма и из укороченных векторных волновых уравнений, дают одинаковые результаты, совпадающие с результатами модового подхода. Объяснены причины различия ранее полученных результатов.
\end{abstract}

Ключевые слова: оптический эффект Магнуса, циркулярная поляризация, принцип Ферма, вектор Пойнтинга.

\section{1. ВВЕДЕНИЕ}

В настоящее время в различных областях физики интенсивно исследуются эффекты, связанные с поляризацией частиц. Например, в ядерных взаимодействиях учет поляризации частиц позволяет проанализировать нарушения пространственной четности при радиационном захвате нейтронов [1] и в упругом канале взаимодействия нейтронов с ядрами [2], спиновые эффекты в физике высоких энергий [3] и при делении ядер [4], а также поляризационные эффекты в оптике.

Среди поляризационных эффектов в оптике, вызванных взаимным влиянием параметров траектории и поляризации, следует назвать закон параллельного переноса Рытова, приводящий к вращению плоскости поляризации поля относительно естественного трехгранника Френе (см. [5], с. 403, или [6], с. 221). Экспериментально это явление наблюдалось при прохождении линейно поляризованного света через спирально скрученное одномодовое волокно [7]. Фаза Рытова-Владимирского является прототипом фаз Берри и Панчаратнама [8] (с большим обзором работ в этой области можно ознакомиться в [9], [10]). При прохождении циркулярно поляризованного излучения через скрученный многомодовый световод наблюдается не только поворот

*Российский федеральный ядерный центр, Всероссийский научно-исследовательский институт технической физики им. акад. Е. И. Забабахина, Снежинск, Челябинская обл., Россия. E-mail: n.r.sadykov@rambler.ru

3 Теоретическая и математическая физика, т. 149, № 1, 2006 г. 
плоскости поляризации, но и поворот поперечной структуры луча (поворот спеклкартины на выходе из волокна) при изменении шага спирали [11]-[13], причем угол поворота поперечной структуры спекл-картины равен фазе Рытова-Владимирского.

Анализ влияния параметров траектории фотона на его поляризацию дает основание ожидать обратный эффект - влияние поляризации (спина фотона) на процесс распространения фотона. На основе этой гипотезы были предсказаны и экспериментально установлены оптический эффект Магнуса [14]-[16]; влияние продольного магнитного поля на параметры излучения в волокне, проявляющееся в повороте спекл-картины на выходе из волокна при смене направления магнитного поля [17]; взаимное влияние параметров поляризации и траектории в однородной изотропной среде [18], а именно поперечное отклонение продольной компоненты пучка лучей вдоль оси $O X$ при формировании с помощью линзы асимметричного вдоль оси $O Y$ волнового фронта; поворот поперечной структуры луча (поворот спекл-картины на выходе из волокна) при изменении шага спирали траектории [11]-[13] на величину угла, равную фазе Рытова-Владимирского и ряд других эффектов.

В [14]-[16] теоретическое обоснование оптического эффекта Магнуса было получено в рамках волновой оптики в терминах волноводных мод. Волноводная оптика является той самой областью, в которой эволюцию электромагнитного излучения можно описать как в рамках волноводных мод, так и в рамках геометрической оптики. Оба эти подхода, взаимно дополняя друг друга, позволяют описать одни и те же эффекты. В приближении геометрической оптики оптический эффект Магнуса, как и в случае волновой оптики, перекликается с законом параллельного переноса Рытова. В приближении геометрической оптики эти два эффекта являются взаимно обратными: оптический эффект Магнуса (точнее, оптический эффект пинг-понга) приводит к дополнительному кручению искривленной траектории [19]-[25], а кручение, которое неразрывно связано с законом параллельного переноса Рытова, т.е. с фазой Берри, приводит к изменению кривизны траектории фотона [11]-[13]. На данный момент времени известно три метода вывода уравнений траектории пучка лучей, описывающих оптический эффект Магнуса: на основе канонических уравнений Гамильтона с феноменологически полученным гамильтонианом, из укороченного действия и из укороченных векторных волновых уравнений. Из опубликованных в работах [11], [19], [24] результатов следует, что величины вращения спекл-картины на выходе из волокна, полученные в рамках методов укороченного действия и укороченных векторных волновых уравнений, совпадают и оказываются в два раза меньше, чем в случае вывода уравнения из канонических уравнений Гамильтона. В данной работе показано, что все три метода дают одинаковые результаты, которые совпадают с результатами модового подхода [15].

\section{2. ВЫВОД УРАВНЕНИЯ ТРАЕКТОРИИ ПУЧКА ЛУЧЕЙ ИЗ ПРИНЦИПА ФЕРМА (УКОРОЧЕННОГО ДЕЙСТВИЯ)}

В этом разделе мы получим из принципа Ферма уравнение траектории пучка лучей, описывающее оптический эффект Магнуса в приближении геометрической 
оптики в слабо неоднородной локально изотропной среде. Для этого воспользуемся результатами работы [24]

Рассмотрим влияние изгибов волоконного световода с нулевым кручением на параметры пробной функции направляемых мод [23], [24], считая кривизну изгиба малой $(R \gg \widetilde{\lambda}$, где $R$ - радиус кривизны, $\widetilde{\lambda}$ - длина волны де Бройля). В качестве пробной функции используем функцию вида

$$
\begin{gathered}
\mathbf{E}(r, \varphi)=\frac{\mathbf{e}_{x}+i \sigma \mathbf{e}_{y}}{\sqrt{2}} \Psi(r, \varphi, z), \\
\Psi(r, \varphi)=\Psi_{0}(r) \exp \left[r(\kappa \cos \varphi+i \eta \sin \varphi)+i \beta_{1} z\right],
\end{gathered}
$$

где $\beta_{1}=$ const, $\Psi_{0}(r)$ - решение скалярного волнового уравнения

$$
\frac{\partial^{2} \Psi_{0}}{\partial r^{2}}+\frac{1}{r} \frac{\partial}{\partial r} \Psi_{0}+n^{2}(r) k^{2} \Psi_{0}=\beta_{0}^{2} \Psi_{0} .
$$

Здесь $(r, \varphi, z)$ - координаты тороидальной системы координат (коэффициенты Ламе равны $h_{1}=1, h_{2}=r, h_{3}=1+r \cos \varphi / R$ [24]): $z$ - координата, отсчитываемая вдоль оси симметрии, $\varphi$ - угол между кривизной и радиусом $\mathbf{r}$ в локально цилиндрической системе координат, центр которой лежит на оси световода; $\kappa, \eta$ - параметры пробной функции, $\sigma= \pm 1$ - знак циркулярной поляризации, $\beta_{0}=$ const, $k=2 \pi / \lambda, \lambda-$ длина волны в вакууме, $n=n(r)$ - показатель преломления световода. Нетрудно заметить, что при $R \rightarrow \infty$ тороидальная система координат переходит в цилиндрическую. Поэтому в дальнейшем, чтобы не вводить дополнительных обозначений, мы будем использовать те же переменные $r, \varphi, z$ в качестве координат цилиндрической системы, но при этом обязательно указывать, какая система координат используется.

Учет поляризационного слагаемого приводит к тому, что помимо ранее известного смещения амплитуды моды вдоль радиуса кривизны (см. книгу [26], с. 410) постоянная распространения пробной функции направляемой моды содержит бинормальную компоненту, а параметры пробной функции, как и в случае скалярного волнового уравнения (без поляризационного слагаемого), удовлетворяют вариационному принципу (см. книгу [26], с. 289)

$$
\beta_{1}^{2}=\beta_{0}^{2}-\frac{1}{2}\left(\kappa-2 \frac{a^{2} \beta_{0}^{2}}{R}\right)^{2}-\frac{1}{2}\left(\eta+\frac{\sigma}{2 R}\right)^{2}+o\left(\frac{1}{R^{2}}\right),
$$

где $a$ - среднеквадратичный размер пятна моды. В соответствии с этим принципом, если пробная функция является хорошим приближением решения волнового уравнения, то ее параметры будут соответствовать наибольшему значению $\beta$. Вариационный принцип в нерелятивистской квантовой механике означает, что полная энергия частицы в квантовом состоянии должна быть минимальна, и это является естественным требованием. Из (2) в соответствии с вариационным принципом получим [24]

$$
\kappa=\frac{2 a^{2} \beta_{0}^{2}}{R}, \quad \eta=-\frac{\sigma}{2 R} .
$$


Воспользуемся этими соотношениями для вывода в приближении геометрической оптики уравнений траектории луча в неоднородной локально изотропной среде и применим полученные уравнения для описания оптического эффекта Магнуса. Предположим, что показатель преломления изменяется достаточно мало на расстояниях порядка длины волны (приближение геометрической оптики), а роль собственной функции играет амплитуда волны, которая также медленно меняется с расстоянием, и волна остается "почти плоской". На основании такой модели с учетом поперечной компоненты у волнового числа (постоянной распространения) укороченное действие для фотона запишется как

$$
S_{0}=\int_{1}^{2}\left(\beta d s+A_{\alpha} d x^{\alpha}\right),
$$

где $\beta=n k, s-$ натуральный параметр. В соответствии с (3)

$$
\mathbf{A}=\frac{\sigma}{2} \mathbf{l} \times \vec{\nabla} \ln n, \quad \frac{\mathbf{P}}{\hbar}=\frac{\mathbf{p}}{\hbar}+\mathbf{A}, \quad \mathbf{p}=n k \mathbf{l},
$$

где $\mathbf{P}$ - обобщенный импульс фотона, $\mathbf{l}$ - касательный к траектории единичный вектор. Видно, что обобщенный импульс фотона содержит бинормальную к траектории компоненту. Нетрудно заметить, что действие (4) совпадает с действием частицы в магнитном поле, где вектор А играет роль вектора электромагнитного поля.

Приравняв вариацию от действия (4) нулю, получим уравнение траектории пучка лучей в неоднородной локально изотропной среде [12]

$$
\frac{\partial \mathbf{l}}{\partial s}=\mathbf{l} \times \vec{\nabla} \ln n \times \mathbf{l}+\frac{1}{k n} \mathbf{l} \times \operatorname{rot} \mathbf{A} .
$$

В работах [23], [24] второе слагаемое в (6) мы записали в виде $(\mathbf{l} \times \mathbf{l} \times \partial \mathbf{A} / \partial s) /(k n)$, т.е. пренебрегли поперечными компонентами ротора. Как будет показано ниже, для световода с параболическим профилем показатель преломления (ППП) в цилиндрической системе координат эта величина в два раза меньше, чем $(\mathbf{l} \times \operatorname{rot} \mathbf{A}) /(k n)$. Это приводит к тому, что аналитически вычисленная величина поворота спекл-картины на выходе из волокна при смене знака циркулярной поляризации оказывается в два раза меньше, чем при модовом подходе [15], [16].

Применим уравнение (6) к описанию оптического эффекта Магнуса в слабоградиентном волокне (световоде с параболическим ППП) и в сильноградиентном волокне (световоде со ступенчатым ППП).

Рассмотрим световод с неограниченным параболическим ППП

$$
n^{2}=n_{\mathrm{co}}^{2}\left(1-2 \Delta\left(\frac{r}{\rho}\right)^{2}\right),
$$

где $\Delta \ll 1, n_{\text {со }}=$ const, $\rho=$ const (в случае световода с усеченным ППП $\rho$ играет роль сердцевины). 
Из (6), (7) в цилиндрических координатах $r, \varphi, z$ получим [27]

$$
\begin{gathered}
\frac{d \mathbf{l}}{d s}=\mathbf{e}_{r}\left[r_{s s}^{\prime \prime}-r\left(\varphi_{s}^{\prime}\right)^{2}\right]+\mathbf{e}_{\varphi}\left[r \varphi_{s s}^{\prime \prime}+2 r_{s}^{\prime} \varphi_{s}^{\prime}\right]+\mathbf{e}_{z} z_{s s}^{\prime \prime}, \\
\mathbf{l} \times \vec{\nabla} \ln n \times \mathbf{l}=\mathbf{e}_{r} \frac{\partial \ln n}{\partial r}-\left(\mathbf{e}_{r} r_{s}^{\prime}+\mathbf{e}_{\varphi} r \varphi_{s}^{\prime}+\mathbf{e}_{z} z_{s}^{\prime}\right) \frac{\partial \ln n}{\partial s}, \\
\mathbf{A}=-\frac{\sigma}{2}\left(\mathbf{e}_{z} r \varphi_{s}^{\prime}-\mathbf{e}_{\varphi}\right) \frac{\partial \ln n}{\partial r}, \quad \operatorname{rot} \mathbf{A}=\frac{\sigma}{2 r}\left[\mathbf{e}_{\varphi} r \varphi_{s}^{\prime}+\mathbf{e}_{z}\right] \frac{\partial}{\partial r}\left(r \frac{\partial \ln n}{\partial r}\right),
\end{gathered}
$$

где $\mathbf{r}=\mathbf{e}_{r} r+\mathbf{e}_{z} z, \mathbf{l}=d \mathbf{r} / d s=\mathbf{e}_{r} r_{s}^{\prime}+\mathbf{e}_{\varphi} r \varphi_{s}^{\prime}+\mathbf{e}_{z} z_{s}^{\prime} ; \quad r_{s}^{\prime}, \varphi_{s}^{\prime}$ и $z_{s}^{\prime}-$ соответственно производные $r, \varphi$ и $z$ по $s$. Здесь в отличие от [27] вместо $\mathbf{l} \times \partial \mathbf{A} / \partial s$ мы использовали $\operatorname{rot} \mathbf{A}$, и это в соответствии с (8) для световода с параболическим ППП приводит к тому, что компонента вектора $(\mathbf{l} \times \operatorname{rot} \mathbf{A})$, направленная вдоль $\mathbf{e}_{\varphi}$, будет в два раза больше соответствующей компоненты вектора $(\mathbf{1} \times \mathbf{1} \times \partial \mathbf{A} / \partial s)$.

Подставим (8) в (6) и выделим азимутальную компоненту. В результате для световода с параболическим ППП (7) с учетом соотношения $\partial(r \partial \ln n / \partial r) / \partial r=-4 r \Delta / \rho^{2}$ получим

$$
n_{\mathrm{co}} r^{2} \varphi_{s}^{\prime}=l_{0}+\frac{\sigma}{k} \frac{\Delta}{\rho^{2}} r^{2},
$$

где $l_{0}=$ const. Выражение (9) представляет собой интеграл движения - закон сохранения проекции орбитального момента на ось волокна. В [21] этот интеграл был получен с помощью канонических уравнений Гамильтона в слабонаправляющем волокне для параксиальных лучей.

Равенство (9) означает, что разность углов поворота $\Delta \varphi=\varphi^{+}-\varphi^{-}$пучка лучей вдоль оси световода равна $\Delta \varphi=2 \Delta /\left(n_{\mathrm{co}} k \rho^{2}\right) z$. В случае оптического эффекта Магнуса это означает, что полученные в [24] из укороченного действия (в приближении геометрической оптики) результаты для световода с параболическим ППП совпадают с результатами волнового метода (модового подхода [15]).

Из (8) следует, в случае световода с параболическим ППП азимутальная компонента вектора $\mathbf{l} \times \operatorname{rot} \mathbf{A}$ в два раза больше азимутальной компоненты вектора $(\mathbf{l} \times \mathbf{l} \times \partial \mathbf{A} / \partial s)$. Именно этим объясняется отличие аналитических результатов работы [24] от экспериментальных результатов [15], [16].

\section{3. ВЫВОД УРАВНЕНИЯ ТРАЕКТОРИИ ПУЧКА ЛУЧЕЙ ИЗ КАНОНИЧЕСКИХ УРАВНЕНИЙ ГАМИЛЬТОНА}

В работе [20] результаты анализа бокового смещения при преломлении луча на границе раздела двух однородных изотропных сред с различными показателями преломления были обобщены на слабонеоднородную среду и феноменологически получено уравнение поперечного смещения луча

$$
\frac{d\left(\Delta \mathbf{r}_{\perp}\right)}{d s}=-\sigma \frac{1}{k n}(\tilde{\mathbf{l}} \times \vec{\nabla} \ln n)
$$

где $\tilde{\mathbf{l}}$ - параллельный волновому вектору единичный вектор. Это уравнение позволило авторам работы [20] "модифицировать" основные уравнения геометрической 
оптики:

$$
\frac{d \mathbf{r}}{d s}=\tilde{\mathbf{l}}-\sigma \frac{1}{k n}(\tilde{\mathbf{l}} \times \vec{\nabla} \ln n), \quad \frac{d \tilde{\mathbf{l}}}{d s}=\vec{\nabla} \ln n-\tilde{\mathbf{l}}(\tilde{\mathbf{l}} \vec{\nabla} \ln n) .
$$

Первое из этих уравнений влечет, что угол между $\tilde{\mathbf{l}}$ и единичным касательным к траектории вектором $d \mathbf{r} / d s$ не равен нулю.

Если (11) рассматривать как канонические уравнения Гамильтона [17], то в этом случае гамильтониан $H$ равен [20]

$$
H(\mathbf{P}, \mathbf{u}, \mathbf{r}, \mathbf{e})=\frac{c}{n(\mathbf{r})} P-\frac{c^{2}}{n^{2} \hbar \omega} \mathbf{P} \cdot[(\mathbf{e} \times \mathbf{u}) \times \vec{\nabla} \ln n]=\frac{c}{n} P-\frac{\sigma c}{n^{2} k} \mathbf{P} \cdot[\tilde{\mathbf{l}} \times \vec{\nabla} \ln n],
$$

где $k=\omega / c, \mathbf{r}, \mathbf{e}$ - обобщенные координаты, $\mathbf{r}$ характеризует положение точки на траектории, $\mathbf{l}$ - трехмерный вектор поляризации; $\mathbf{P}, \mathbf{u}$ - обобщенные импульсы, вектор $\mathbf{P}$ определен в (5), $\mathbf{u}=i \hbar \mathbf{e}^{*}$ (* означает комплексное сопряжение). С точностью до величин, имеющих первый порядок по кривизне, $\mathbf{e} \times \mathbf{u}=\sigma \tilde{\mathbf{l}}$. Видно, что гамильтониан (12) зависит только от показателя преломления и не зависит от импеданса. При этом второе слагаемое в каждой точке траектории с точностью до величин первого порядка по кривизне равно нулю и, таким образом, удовлетворяет требованиям закона сохранения энергии в случае укороченного действия. Для гамильтониана (12) с учетом условий ортогональности $\mathbf{e P}=\mathbf{u P}=0$ вектора поляризации и обобщенного импульса в каждой точке траектории верны канонические уравнения (11), а также уравнение параллельного переноса Рытова

$$
\frac{d \mathbf{e}}{d s}=-\tilde{\mathbf{l}}(\mathbf{e} \cdot \vec{\nabla} \ln n) .
$$

Из (11) и (13) следует условие поперечности: $\mathbf{e} \tilde{\mathbf{l}}=0$.

Авторы работы [20] указывали как на досадное недоразумение на то, что из первого уравнения (11) следует $|d \mathbf{r} / d s| \neq 1$. В действительности никакого недоразумения здесь нет. В данной работе альтернативными методами мы получим уравнения траектории пучка лучей, из которых следует, что уравнения траектории (11) справедливы с точностью до первого порядка радиуса кривизны. Поэтому в (11) из $|\tilde{\mathbf{l}}|=1$ следует, что $|d \mathbf{r} / d s|=1$. Более того, уравнения (11) выводятся из канонических уравнений Гамильтона на основе гамильтониана (12), и это означает, что касательным к траектории вектором будет $d \mathbf{r} / d s$, который обязан иметь единичную длину, $|d \mathbf{r} / d s|=1$, причем вектор $\tilde{\mathbf{l}}$ в (11) определяет направление обобщенного импульса P.

Гораздо бо́льшая проблема феноменологически полученных уравнений (11) состоит в том, что они дают в два раза большее значение для обобщенного импульса $\mathbf{P}$, чем в случае вывода уравнения пучка лучей из принципа Ферма (см. раздел 2). Действительно, из первого уравнения (11) следует, что

$$
\mathbf{l}=\frac{d \mathbf{r}}{d s}, \quad \mathbf{p}=\hbar n k \mathbf{l}, \quad \mathbf{P}=\mathbf{p}+2 \hbar \mathbf{A}, \quad \mathbf{A}=\frac{\sigma}{2}(\mathbf{l} \times \vec{\nabla} \ln n),
$$

где мы отбросили пропорциональные квадрату кривизны слагаемые. Видно, что второе слагаемое в выражении для обобщенного импульса $\mathbf{P}$ в два раза больше аналогичной величины в (5). Но из (5) следует уравнение (6), которое в приближении 
геометрической оптики в световоде с параболическим ППП дает ту же величину поворота спекл-картины в оптическом эффекте Магнуса, что и в случае модового подхода [15]. Аналогичные результаты следуют из рассуждений, приведенных в разделе 4 данной работы, где уравнение траектории пучка лучей получено из укороченных векторных волновых уравнений. Таким образом, из гамильтониана (12) для оптического эффекта Магнуса в приближении геометрической оптики получается в два раза бо́льшая величина поворота спекл-картины, чем при модовом подходе, и для совпадения результатов нужно второе слагаемое в (12) уменьшить в два раза.

Покажем еще одним способом, что гамильтониан (12) для оптического эффекта Магнуса дает по сравнению с модовым подходом в два раза больший результат. В нерелятивистском случае переход к новой системе координат, которая движется со скоростью V относительно лабораторной системы координат, равносилен преобразованию гамильтониана (см. книгу [28], с. 307)

$$
H^{\prime}=H-\mathbf{V} \cdot \mathbf{P}
$$

При нерелятивистской скорости $V \ll c$ последнее слагаемое представляет собой релятивистское преобразование нулевой компоненты 4-импульса: $E^{\prime}=\gamma(E-\mathbf{V} \cdot \mathbf{P})$, $\gamma=1 / \sqrt{1-V^{2} / c^{2}},|\mathbf{V}|=V$. Таким образом, представление гамильтониана в виде (12) позволяет утверждать, что для данной лабораторной системы отсчета $K$ существует другая система отсчета $K^{\prime}$, в которой гамильтониан не содержит второе слагаемое в правой части (12), т.е. гамильтониан в системе $K^{\prime}$ равен $H^{\prime}=c P / n$. Нетрудно заметить, что система $K^{\prime}$ должна двигаться относительно $K$ со скоростью $\mathbf{V}_{0}$ такой, что

$$
\mathbf{V}_{0}=-\frac{\sigma c}{n^{2} k} \tilde{\mathbf{l}} \times \vec{\nabla} \ln n .
$$

В случае параболического ППП $n^{2}=n_{\text {со }}^{2}\left(1-2 \Delta(r / \rho)^{2}\right), \Delta \ll 1$, из последнего равенства следует, что

$$
\Omega=\frac{d \varphi}{d t}=2 \frac{\sigma c}{n^{2} k} \cdot \frac{\Delta}{\rho^{2}}
$$

где $\vec{\Omega} \| \tilde{\mathbf{l}}, \mathbf{V}_{0}=\vec{\Omega} \times \mathbf{r}, \mathbf{r}-$ радиус-вектор в цилиндрической системе координат $(r, \varphi, z)$. Относительно приращения натурального параметра $d s=(c / n) d t$ последнее равенство запишется как

$$
\frac{d \varphi}{d s}=2 \frac{\sigma}{n k} \cdot \frac{\Delta}{\rho^{2}}
$$

при этом его правая часть в два раза больше, чем соответствующее слагаемое в (9).

Авторы работы [20] применили уравнения (11) для описания оптического эффекта Магнуса в многомодовом световоде с параболическим ППП. Из результатов [20] следует, что в зависимости от знака циркулярной поляризации лучи будут поворачиваться относительно продольной оси в противоположные стороны. Величина угла поворота спекл-картины при смене знака циркулярной поляризации при этом равна $\Delta \varphi=2 \Delta /\left(n_{\text {со }} k \rho^{2}\right) z$, что совпадает с результатом волнового подхода. В чем причина такого несоответствия результатов работы [20] с приведенными выше результатами? Дело в том, что из гамильтониана (12) следует, что второе уравнение 
системы (11) записывается в виде $d \mathbf{P} / d t=-\vec{\nabla} H(\mathbf{P}, \mathbf{r})$, что равносильно

$$
\frac{d \mathbf{p}}{d t}+2 \hbar \frac{d \mathbf{A}(\mathbf{r})}{d t}=\mathbf{l} \times \vec{\nabla} \ln n \times \mathbf{p}+\frac{2 c}{n^{2} k} \vec{\nabla}(\mathbf{p A}) .
$$

Учитывая, что А явно не зависит от времени, преобразуем второе слагаемое в левой части последнего равенства:

$$
\frac{d \mathbf{A}}{d t}=\frac{c}{n}(\mathbf{l} \vec{\nabla}) \mathbf{A}=\frac{c}{n^{2} k \hbar}(\mathbf{p} \vec{\nabla}) \mathbf{A} .
$$

По аналогии с выводом уравнения траектории заряженной частицы получим уравнение траектории пучка лучей:

$$
\frac{d \mathbf{l}}{d s}=\frac{c}{n} \cdot \frac{d \mathbf{l}}{d t}=\mathbf{l} \times \vec{\nabla} \ln n \times \mathbf{l}+\frac{2}{n k} \mathbf{l} \times \operatorname{rot} \mathbf{A},
$$

в котором второе слагаемое в правой части в два раза больше, чем в уравнении (6).

\section{4. ОПТИЧЕСКИЙ ЭФФЕКТ МАГНУСА В ПРИБЛИЖЕНИИ ГЕОМЕТРИЧЕСКОЙ ОПТИКИ В СИЛЬНОГРАДИЕНТНОМ ВОЛОКНЕ}

В этом разделе мы применим полученные в разделах 2, 3 результаты к световоду с сильноградиентным (ступенчатым) ППП по аналогии с работой [20].

В работе [20] уравнения (11) применены для описания в приближении геометрической оптики оптического эффекта Магнуса в сильноградиентном волокне (световоде со ступенчатым ППП). При этом было сделано следующее предположение о поведении показателя преломления на границе раздела двух неоднородных локально изотропных сред: в некотором интервале $[0, h]$ значений координаты, отсчитываемой перпендикулярно границе раздела, показатель преломления меняется по параболическому закону от $n_{1}$ до $n_{2}=n_{1}-\Delta n, \Delta n \ll 1$. Далее вычислялся угол отклонения луча в поперечном направлении в точке полного отражения в соответствии с первым уравнением в (11), что для световода с цилиндрической симметрией приводит к вращению пучка лучей вдоль оси волокна. Если величину поворота за один акт умножить на число отражений на длине волокна, то мы получим величину угла поворота пучка лучей относительно оси симметрии волокна, при этом число отражений в волокне определяется из второго уравнения в (11). В соответствии с этой моделью угол поворота спекл-картины в сильноградиентном волокне равен углу поворота спекл-картины в световоде с параболическим ППП: $\Delta \varphi=2 \Delta /\left(n_{\mathrm{co}} k \rho^{2}\right) z$, что приблизительно в два раза больше, чем было получено в эксперименте [15], [16]. Решению этой проблемы посвящена работа [21]. Недостаток этого подхода состоит в том, что ступенчатый профиль аппроксимируется параболической зависимостью.

Применим уравнение (6) к оптическому эффекту Магнуса в случае сильноградиентного волокна. Как указывалось выше, величина угла поворота пучка лучей относительно оси симметрии волокна равна произведению величины поворота за один акт на число отражений на длине волокна. В случае уравнения траектории пучка лучей, полученного из принципа Ферма, угол поворота за один акт отражения 
будет вычисляться в соответствии с уравнением (6). Для световода с сильноградиентным ППП по аналогии с [20] введем профиль ППП $n(x)=n_{1}\left(1-2 \Delta(x / h)^{2}\right)$, где $n_{2}=n(h)=n_{1}(1-2 \Delta)$, ось $O X$ направлена перпендикулярно границе раздела. В дальнейшем ось $O Y$ перпендикулярна оси $O X$ и параллельна границе раздела двух сред, ось $O Z$ перпендикулярна предыдущим двум. Из (5) несложно получить, что $\mathbf{A}=-\mathbf{e}_{x} \sigma x \Delta / h^{2}, \operatorname{rot} \mathbf{A}=-\mathbf{e}_{z} \sigma \Delta / h^{2}, \mathbf{l} \times \operatorname{rot} \mathbf{A}=\mathbf{e}_{y} \sigma(\partial x / \partial s) \Delta / h^{2}$. Подставив полученные равенства в (6), получим уравнение $d^{2} y / d s^{2}=\sigma(\partial x / \partial s) \Delta /\left(n k h^{2}\right)$. Проинтегрировав полученное уравнение с учетом начальных условий, получим уравнение, описывающее единичный акт “отражения” в формализме Лагранжа:

$$
\frac{d y}{d s}=\frac{\sigma x \Delta}{n k h^{2}} .
$$

Нетрудно заметить, что в формализме Гамильтона в соответствии с первым уравнением (11) уравнение отражения луча запишется в виде $d y / d s=2 \sigma x \Delta /\left(n k h^{2}\right)$, т.е. в рамках используемого в этом разделе подхода величина поперечного отклонения при единичном акте отражения (14) в два раза меньше, чем в случае формализма Гамильтона. Поскольку число единичных актов отражения при фиксированной длине не зависит от величины малого поперечного смещения в результате одного акта отражения, то в рамках рассматриваемого подхода величина вращения пучка лучей вокруг оси волокна и, как следствие, вращение спекл-картины на выходе из волокна при смене знака циркулярной поляризации будут в два раза меньше, чем при гамильтоновом подходе, что находится в полном согласии с экспериментом [15], [16]. Решению этой проблемы на основе уравнений Гамильтона частично посвящена работа [21].

\section{5. ВЫВОД УРАВНЕНИЯ ТРАЕКТОРИИ ПУЧКА ЛУЧЕЙ ИЗ УКОРОЧЕННЫХ ВЕКТОРНЫХ ВОЛНОВЫХ УРАВНЕНИЙ}

В предыдущих разделах анализ оптического эффекта Магнуса был проведен в приближении геометрической оптики на основе уравнений, полученных из канонических уравнений Гамильтона и из принципа Ферма (укороченного действия). В этом разделе рассмотрим альтернативный к двум предыдущим подходам метод вывод уравнения траектории луча с учетом спиральности фотона из укороченных векторных волновых уравнений [24], [25], [29]. Вывод уравнения траектории частицы с помощью укороченных волновых уравнений помимо оптического эффекта Магнуса позволяет рассмотреть и предсказать другие эффекты.

Суть подхода состоит в следующем. Из уравнений Максвелла несложно получить укороченные векторные волновые уравнения [19], [25]

$$
\begin{array}{r}
2 \beta \frac{\partial \mathbf{E}_{0}}{\partial s}+\mathbf{E}_{0} \frac{\partial \beta}{\partial s}+\frac{\omega}{c} \vec{\nabla} \mu \times \mathbf{H}_{0}+\beta \mathbf{l}\left(\mathbf{E}_{0} \vec{\nabla} \ln \varepsilon\right)=0, \\
2 \beta \frac{\partial \mathbf{H}_{0}^{*}}{\partial s}+\mathbf{H}_{0}^{*} \frac{\partial \beta}{\partial s}-\frac{\omega}{c} \vec{\nabla} \varepsilon \times \mathbf{E}_{0}^{*}+\beta \mathbf{l}\left(\mathbf{H}_{0}^{*} \vec{\nabla} \ln \mu\right)=0,
\end{array}
$$

где $n^{2}=\varepsilon \mu, \mathbf{E}=\mathbf{E}_{0}(\mathbf{r}) \exp [-i \omega t+i \Psi(\mathbf{r l})], \mathbf{H}=\mathbf{H}_{0}(\mathbf{r}) \exp [-i \omega t+i \Psi(\mathbf{r l})], \quad \beta \mathbf{l}=$ $\operatorname{grad} \Psi(\mathbf{r l})$. 
Укороченные волновые уравнения (15) без учета поляризационных слагаемых (последние слагаемые в каждом из уравнений (15)) представляют собой основные уравнения геометрической оптики, которые могут быть получены разложением по безразмерному параметру, имеющему порядок $1 /(k L)$. В этом случае наиболее последовательный способ вывода этих уравнений геометрической оптики принадлежит Рытову [30], который искал решение скалярного волнового уравнения в виде произведения медленной амплитуды на быстроосциллирующую экспоненту. Полученная таким образом система уравнений включает в себя уравнение эйконала и уравнение переноса излучения, принадлежащее к классу уравнений Гамильтона-Якоби (см. [6], с. 9) и в стационарных условиях сводящееся к принципу Ферма.

Умножив векторно первое уравнение (15) на $\mathbf{H}_{0}^{*}$, а второе - на $\mathbf{E}_{0}$ и вычтя первое уравнение из второго, после несложных преобразований получим (более подробно cм. [19], [25])

$$
\begin{aligned}
2 \beta \frac{\partial \mathbf{S}}{\partial s}+ & 2 \mathbf{S} \frac{\partial \beta}{\partial s}+\frac{c}{16 \pi}\left\{\beta \mathbf{E}_{0} \times \mathbf{l}\left(\mathbf{H}_{0}^{*} \vec{\nabla} \ln \mu\right)-\frac{\omega}{c} \mathbf{H}_{0}^{*} \times \vec{\nabla} \mu \times \mathbf{H}_{0}\right\}- \\
- & \frac{c}{16 \pi}\left[\frac{\omega}{c} \mathbf{E}_{0} \times \vec{\nabla} \varepsilon \times \mathbf{E}_{0}^{*}+\beta \mathbf{H}_{0}^{*} \times \mathbf{l}\left(\mathbf{E}_{0} \vec{\nabla} \ln \varepsilon\right)\right]=0,
\end{aligned}
$$

где $\mathbf{S}=(c / 16 \pi)\left\{\mathbf{E}_{0} \times \mathbf{H}_{0}^{*}+\right.$ к.с. $\}$ - вектор Пойнтинга. Выражение в фигурных скобках в (16) с учетом уравнений Максвелла в приближении геометрической оптики можно записать как $\beta \vec{\nabla} \ln \mu\left(\mathbf{l} \cdot \mathbf{H}_{0}^{*} \times \mathbf{E}_{0}\right)$, а в квадратных скобках - как $\beta \vec{\nabla} \ln \varepsilon\left(\mathbf{l} \cdot \mathbf{H}_{0}^{*} \times \mathbf{E}_{0}\right)$. Окончательно уравнение (16) запишется следующим образом:

$$
\frac{\partial \mathbf{S}}{\partial s}=\mathbf{1} \times \vec{\nabla} \ln n \times \mathbf{S} .
$$

В (15) последние слагаемые в первом и втором уравнениях представляют собой соответственно выражения $\vec{\nabla}(\mathbf{E} \vec{\nabla} \ln \varepsilon)$ и $\vec{\nabla}\left(\mathbf{H}_{0}^{*} \vec{\nabla} \ln \mu\right)$, которые обуславливают поляризационные эффекты. При выводе (17) были отброшены слагаемые, пропорциональные $-i \vec{\nabla}\left(\mathbf{E}_{0} \vec{\nabla} \ln \varepsilon\right)$ и $i \vec{\nabla}\left(\mathbf{H}_{0}^{*} \vec{\nabla} \ln \mu\right)$. В дальнейшем в соответствии с работой [19] будем учитывать только те слагаемые, которые параллельны 1. Тогда дополнительные слагаемые в левых частях первого и второго уравнений (15) будут равны соответственно

$$
-i \mathbf{l} \frac{\partial}{\partial s}\left(\mathbf{E}_{0} \vec{\nabla} \ln \varepsilon\right), \quad i \mathbf{l} \frac{\partial}{\partial s}\left(\mathbf{H}_{0}^{*} \vec{\nabla} \ln \mu^{*}\right)
$$

а дополнительное слагаемое в (16) (более подробно см. [19], [24]) -

$$
\begin{array}{r}
\frac{i \omega}{16 \pi \beta}\left[-\varepsilon \vec{\nabla} \frac{\partial \ln \varepsilon}{\partial s} \times \mathbf{E}_{0} \times \mathbf{E}_{0}^{*}-\mu \vec{\nabla} \frac{\partial \ln \mu}{\partial s} \times \mathbf{H}_{0} \times \mathbf{H}_{0}^{*}-\right. \\
\left.-\frac{1}{2} \vec{\nabla} \varepsilon \times \frac{\partial}{\partial s}\left(\mathbf{E}_{0} \times \mathbf{E}_{0}^{*}\right)-\frac{1}{2} \vec{\nabla} \mu \times \frac{\partial}{\partial s}\left(\mathbf{H}_{0} \times \mathbf{H}_{0}^{*}\right)\right],
\end{array}
$$

где $\omega=2 \pi c / \lambda$. Последнее выражение показывает, что добавка к уравнению траектории луча (16) зависит от знака циркулярной поляризации луча, поскольку наличие циркулярной поляризации $\mathbf{E}_{0}$, пропорциональной $\left(\mathbf{e}_{1}+i \sigma \mathbf{e}_{2}\right) / \sqrt{2}$, означает, что 
$\mathbf{E} \times \mathbf{E}^{*} \sim \sigma \mathbf{S}, \mathbf{H} \times \mathbf{H}^{*} \sim \sigma \mathbf{S}, \mathbf{H}_{0} \sim \mathbf{l} \times \mathbf{E}_{0}, \mathbf{e}_{1} \perp \mathbf{e}_{2},\left|\mathbf{e}_{1}\right|=\left|\mathbf{e}_{2}\right|=1$. На основании уравнений Максвелла нетрудно получить следующие равенства:

$$
\frac{c}{4 \pi} \mathbf{E}_{0} \times \mathbf{E}_{0}^{*}=-2 i \sigma \frac{\mu \omega}{c \beta} \mathbf{S}, \quad \frac{c}{4 \pi} \mathbf{H}_{0} \times \mathbf{H}_{0}^{*}=-2 i \sigma \frac{\varepsilon \omega}{c \beta} \mathbf{S} .
$$

С учетом (19) и (20) уравнение траектории луча окончательно запишется в виде (более подробно см. [19])

$$
\frac{\partial \mathbf{S}}{\partial s}=\mathbf{l} \times \vec{\nabla} \ln n \times \mathbf{S}+\frac{\sigma}{4 \beta}\left[\frac{\partial}{\partial s}\left(\vec{\nabla} \ln n^{2}\right) \times \mathbf{S}+\frac{1}{2} \vec{\nabla} \ln n^{2} \times \frac{\partial \mathbf{S}}{\partial s}+\frac{1}{\kappa^{2}} \mathbf{S} \times\left(\frac{\partial k}{\partial s} \vec{\nabla} \kappa\right)\right],
$$

где $\mathbf{S}=S \mathbf{l}, \kappa=\mu / \varepsilon-$ импеданс.

Видно, что при $k=0, \partial \mathbf{S} / \partial s=0$ уравнение (21) совпадает с уравнением (6), если последнее слагаемое в (6) записать в виде $(\mathbf{l} \times \mathbf{l} \times \partial \mathbf{A} / \partial s) /(k n)$, т.е. пренебречь поперечными компонентами вектора $\operatorname{rot} \mathbf{A}$. Такая замена в цилиндрической системе координат, как было показано в разделе 2, в световоде с параболическим ППП дает в два раза меньшую величину поворота спекл-картины на выходе из волокна при смене знака циркулярной поляризации, чем величина $(\mathbf{l} \times \operatorname{rot} \mathbf{A}) /(k n)$ в уравнении (6). Это в свою очередь будет означать, что аналитически вычисленная в соответствии с (21) величина поворота спекл-картины на выходе из волокна будет в два раза меньше результата эксперимента [15], [16]. Отличие уравнения (21) от уравнения (6) при $\kappa=0, \partial \mathbf{S} / \partial s=0$ объясняется тем, что при выводе (21) мы пренебрегли поперечными компонентами поляризационных слагаемых в (17). Если этого не делать, поправки (18) к левым частям уравнений (15) нужно заменить на

$$
\begin{aligned}
\mathbf{M} & =-i \vec{\nabla}\left(\mathbf{E}_{0} \vec{\nabla} \ln \varepsilon\right)=-i \vec{\nabla}\left(k \mathbf{H}_{0} \mathbf{l} \times \vec{\nabla} \ln \varepsilon\right), \\
\mathbf{N} & =i \vec{\nabla}\left(\mathbf{H}_{0}^{*} \vec{\nabla} \ln \mu\right)=-i \vec{\nabla}\left(k^{-1} \mathbf{E}_{0}^{*} \mathbf{l} \times \vec{\nabla} \ln \mu\right) .
\end{aligned}
$$

При этом поправка (19) к уравнению (16) принимает вид

$$
\frac{c}{16 \pi}\left\{\mathbf{E}_{0} \times \mathbf{N}-\mathbf{H}_{0}^{*} \times \mathbf{M}\right\}+\text { K.c. }
$$

Учитывая хорошо известное соотношение

$$
\mathbf{a} \times \vec{\nabla}(\mathbf{b c})=\mathbf{a} \times \mathbf{c} \times \operatorname{rot} \mathbf{b}+\mathbf{a} \times \mathbf{b} \times \operatorname{rot} \mathbf{c}+\mathbf{a} \times(\mathbf{c} \vec{\nabla}) \mathbf{b}+\mathbf{a} \times(\mathbf{b} \vec{\nabla}) \mathbf{c},
$$

пренебрегая в $(22)$ импедансом и изменением амплитуд мод $\mathbf{E}_{0}, \mathbf{H}_{\mathbf{0}}$, получим

$$
\begin{aligned}
\frac{c}{16 \pi} \mathbf{E}_{0} \times \mathbf{N} & =\frac{c}{16 \pi}\left\{-i \kappa^{-1} \mathbf{E}_{0} \times \mathbf{E}_{0}^{*} \times \operatorname{rot}(\mathbf{l} \times \vec{\nabla} \ln \mu)-i \kappa^{-1} \mathbf{E}_{0} \times\left(\mathbf{E}_{0}^{*} \vec{\nabla}\right)(\mathbf{l} \times \vec{\nabla} \ln \mu)\right\}, \\
\frac{c}{16 \pi} \mathbf{H}_{0}^{*} \times \mathbf{M} & =\frac{c}{16 \pi}\left\{i \kappa \mathbf{H}_{0}^{*} \times \mathbf{H}_{0} \times \operatorname{rot}(\mathbf{l} \times \vec{\nabla} \ln \varepsilon)-i \kappa \mathbf{E}_{0} \times\left(\mathbf{H}_{0} \vec{\nabla}\right)(\mathbf{l} \times \vec{\nabla} \ln \varepsilon)\right\} .
\end{aligned}
$$

С учетом (25) и (20) выражение (23) запишется как

$$
\frac{c}{16 \pi}\left\{\mathbf{E}_{0} \times \mathbf{N}-\mathbf{H}_{0}^{*} \times \mathbf{M}\right\}+\text { K.c. }=-\frac{\sigma}{2} \mathbf{S} \times \operatorname{rot}\left(\mathbf{l} \times \vec{\nabla} \ln n^{2}\right),
$$


где вторые слагаемые в правых частях уравнений (25) дают вклады, пропорциональные $(\mathbf{l} \times \vec{\nabla}) \times(\mathbf{l} \times \vec{\nabla}) \ln n$, и поэтому мы ими пренебрегаем.

Поскольку (23) является поправкой к левой части (16), то, пренебрегая вторым слагаемым в квадратных скобках в формуле (21), вместо уравнения (21) без учета импеданса окончательно получим

$$
\frac{\partial \mathbf{S}}{\partial s}=\mathbf{l} \times \vec{\nabla} \ln n \times \mathbf{S}+\frac{\sigma}{2 \beta} \mathbf{S} \times \operatorname{rot}(\mathbf{l} \times \vec{\nabla} \ln n) .
$$

Видно, что описывающее оптический эффект Магнуса уравнение (27), полученное с помощью укороченных векторных волновых уравнений, совпадает с полученным из вариационного принципа (укороченного действия) уравнением траектории (6). Это соответственно дает в случае световода с параболическим ППП ту же величину поворота спекл-картины на выходе из волокна при смене знака циркулярной поляризации.

\section{6. СРАВНИТЕЛЬНЫЙ АНАЛИЗ ПОЛУЧЕННЫХ РЕЗУЛЬТАТОВ}

Мы рассмотрели оптический эффект Магнуса в многомодовом световоде с параболическим ППП. Внесены изменения в канонические уравнения Гамильтона, отвечающие уменьшению второго слагаемого в правой части (12) в два раза. Соответствующим образом скорректированные уравнения (6) траектории пучка лучей, полученные из принципа Ферма [23], [24], и уравнения (27), полученные из укороченных векторных волновых уравнений [19], [25], [29], дают одинаковые результаты. Более того, эти результаты совпадают с выводами, полученными в рамках модового подхода. В терминах геометрической оптики оптический эффект Магнуса представляет собой дополнительное кручение траектории фотона при его движении по искривленной траектории переменного радиуса. В случае сильноградиентного волокна (световода со ступенчатым ППП, у которого поведение показателя преломления в приграничной области аппроксимировано параболической зависимостью от поперечной к границе раздела двух сред координаты) скорректированные в этой работе результаты работ [19], [20], [24] хорошо описывают результаты эксперимента [15], причем в отличие от [20] у нас не возникает "проблемы половинки". Следует отметить, что при сравнении с экспериментом в случае сильноградиентного волокна мы рассмотрели только параболическую аппроксимацию зависимости показателя преломления от поперечной координаты и не рассматривали другие функциональные зависимости. Одним из достоинств уравнений, полученных на основе феноменологического гамильтониана, является полевая трактовка действия, согласно которой в качестве переменной величины рассматривается спиновая функция фотона. К сожалению, это направление, насколько это известно автору данной работы, не получило дальнейшего развития.

Уравнения Эйлера-Лагранжа в случае сильно невырожденного лагранжиана эквивалентны каноническим уравнениям Гамильтона в фазовом пространстве [10]. В работах [23], [24] уравнение траектории выводится из принципа Ферма (принципа укороченного действия). Укороченное действие минимально по всем траекториям, 
удовлетворяющим закону сохранению энергии и проходящим через конечную точку в произвольный момент времени. Для того чтобы пользоваться принципом наименьшего действия, необходимо предварительно выразить импульсы через обобщенные координаты и обобщенные скорости с помощью лагранжиана. Малая поправка к феноменологически полученному в [20] гамильтониану, как нетрудно убедиться, принимая во внимание (12), в каждой точке траектории равна нулю. Кроме того, гамильтониан в [20] в соответствии с (12) является однородной функцией первого порядка от импульса: $H(\lambda \mathbf{P}, \mathbf{u}, \mathbf{r}, \mathbf{e})=\lambda H(\mathbf{P}, \mathbf{u}, \mathbf{r}, \mathbf{e}), \lambda>0$. Это означает, что при малых изменениях времени $\mathbf{P} \rightarrow \mathbf{P}^{\prime}=\mathbf{P}-(\partial H / \partial \mathbf{r}) \Delta t, \mathbf{r} \rightarrow \mathbf{r}^{\prime}=\mathbf{r}+(\partial H / \partial \mathbf{P}) \Delta t$ дифференциальная форма $\mathbf{P} d \mathbf{r}$ не меняется [10]. Поэтому эти два внешне различных подхода дают одинаковые результаты для световода с параболическим ППП, отличаясь, однако, по сути. В случае укороченного действия

$$
S_{0}=\int_{1}^{2}\left(\beta d s+A_{\alpha} d x^{\alpha}\right)=\int_{1}^{2} \mathbf{P} \cdot \frac{\partial H}{\partial \mathbf{P}} d t
$$

в работах [23], [24] считается, что вектор скорости $\mathbf{v}=\partial H / \partial \mathbf{P}$ является касательным к траектории, а обобщенный импульс $\mathbf{P}$ в общем случае не параллелен касательному вектору. При этом обобщенный импульс выводится из анализа распространения излучения, проведенного в тороидальной системе координат. Соответственно скорость $d \mathbf{r} / d s$, выраженная через натуральный параметр, в [24] совпадает с единичным касательным вектором l. В уравнении траектории луча (11), полученном в [20] из канонических уравнений Гамильтона, обобщенная скорость также параллельна касательному вектору, но величина $|d \mathbf{r} / d s|$, как отмечают сами авторы работы, не равна единице.

Анализ распространения излучения в тороидальной системе координат показал, что постоянная распространения помимо параметра, характеризующего ранее известный эффект деформации собственной моды вдоль кривизны изогнутого световода [26], содержит бинормальную компоненту [24]. Оба эти параметра удовлетворяют вариационному принципу. По-видимому, параметры, которые характеризуют какие-либо физические процессы, всегда удовлетворяют вариационному принципу.

Вывод уравнения траектории частицы с помощью укороченных векторных волновых уравнений в отличие от других рассматриваемых в работе подходов позволяет рассмотреть и предсказать другие эффекты: обобщение оптического эффекта Магнуса на случай квазимонохроматического излучения [25]; дополнительное скручивание траектории пучка лучей [20], [31] в случае поглощающей среды, причем в градиентном многомодовом поглощающем волокне этот эффект, как и оптический эффект Магнуса, будет приводить к вращению спекл-картины на выходе из волокна, но в отличие от оптического эффекта Магнуса угол вращения спекл-картины будет иметь не линейную, а параболическую зависимость от длины волокна. Учет в работе [25] для квазимонохроматического излучения дисперсионных слагаемых третьего порядка и керровской нелинейности позволяет получить нелинейное уравнение относительно амплитуды вектора Пойнтинга [32]. В случае поглощающей среды уравнение относительно амплитуды вектора Пойнтинга, полученное без учета таких 
слагаемых, представляет собой уравнение Бюргерса [33]. В случае отсутствия поглощения полученное с учетом дисперсионных слагаемых третьего порядка уравнение представляет собой уравнение Кортевега-де Фриза.

В отличие от волнового подхода результаты, полученные в приближении геометрической оптики, могут быть использованы для описания распространения пучка лучей не только в световодах, но и в других неоднородных средах. Световоды в этом случае, как и в случае фазы Берри [7], являются той средой, в которой может быть легко реализован эксперимент для обнаружения оптического эффекта Магнуса.

Полученные в работе результаты частично обобщены на спиновые частицы с ненулевой массой [34].

Благодарности. Автор благодарит Б. Я. Зельдовича за полный список работ с подробным комментарием и интерпретацией полученных в них результатов, а также С. В. Сазонова за обсуждение результатов, ряд полезных замечаний и за моральную поддержку.

\section{Список литературы}

[1] Д. В. Зарецкий, В.К. Сироткин, ЯФ, 39 (1984), 585; E. Kolomensky, V. M. Lobashev, A. V. Pirozhkov et. al., Phys. Lett. B, 107 (1981), 272; Yu. G. Abov, P. A. Krupchitssky, Yu. A. Oratovsky, Phys. Lett. B, 12 (1964), 25; В. М. Лобашев, В. П. Назаренко, П. Ф. Саенко и др., Писъма в ЖЭТФ, 3 (1966), 268.

[2] В.П. Алфименков, УФН, 144 (1984), 361; Л.П. Горьков, И.Е. Дзялошинский, ЖЭЭТФ, 67 (1974), 397.

[3] С. М. Трошин, Н.Е. Тюрин, УФН, 164 (1994), 1073; С. М. Трошин, Н.Е. Тюрин, Спин в физике высоких энергий, Наука, М., 1991.

[4] Г. В. Данилян, УФН, 131 (1980), 329.

[5] Л. Д. Ландау, Е. М. Лифшиц, Электродинамика сплошных сред, Наука, М., 1982.

[6] Ю.А. Кравцов, Ю. И. Орлов, Геометрическая оптика неоднородных сред, Наука, М., 1980.

[7] A. Tomita, R. Y. Chiao, Phys. Rev. Lett., 57 (1986), 937.

[8] M. V. Berry, Proc. Roy. Soc. London. Ser. A, 392 (1984), 45; S. Pancharatnam, Proc. Indian Acad. Sci. Ser. A, 44 (1956), 247.

[9] Д.Н. Клышко, УФН, 163 (1993), 1.

[10] С. И. Виницкий, В. Л. Дербов, В. М. Дубовик и др., УФН, 160:6 (1990), 1.

[11] Н. Р. Садыков, Onm. и спектр., 84:4 (1998), 589.

[12] Н.Р. Садыков, Квантовая электроника, 23 (1996), 277.

[13] Н. Р. Садыков, Известия высших учебных заведений. Физика, 10 (1999), 58.

[14] Б. Я. Зельдович, В. С. Либерман, Квантовая электроника, 17 (1990), 493.

[15] А. В. Дугин, Б. Я. Зельдович, Н. Д. Кундикова, В. С. Либерман, ЖЭТФ, 100 (1991), 1474; A. V. Doogin, N.D. Kundikova, V.S. Liberman, B. Ya. Zeldovich, Phys. Rev. A, 45 (1992), 8204.

[16] А. В. Дугин, Б. Я. Зельдович, Н. Д. Кундикова, В. С. Либерман, Писвма в ЖЭЭФ, 53 (1991), 186.

[17] Н. Б. Баранова, Б.Я. Зельдович, Писъма в ЖЭТФ, 59 (1994), 648; М. Я. Даршт, И. В. Жииргалова, Б.Я. Зельдович, Н.Д. Кундикова, Писъма в ЖЖЭТФ, 59 (1994), 734.

[18] Н. Б. Баранова, А. Ю. Савченко, Б. Я. Зельдович, Писъма в ЖЭЭТФ, 59 (1994), 216; Б. Я. Зельдович, Н.Д. Кундикова, Л.Ф. Рогачева, Писъма в ЖЭТФ, 59 (1994), 737. 
[19] Н. Р. Садыков, Квантовая электроника, 20 (1993), 1137.

[20] V.S. Liberman, B. Ya. Zeldovich, Phys. Rev. A, 46 (1992), 5199.

[21] V.S. Liberman, B. Ya. Zeldovich, Pure and Appl. Optics., 2 (1993), 367.

[22] V.S. Liberman, B. Ya. Zeldovich, Phys. Rev. E, 49 (1994), 2389.

[23] Н.Р. Садыков, Квантовая электроника, 19 (1992), 1021.

[24] Н. Р. Садыков, Квантовал электроника, 20 (1993), 1140.

[25] Н. Р. Садыков, Onm. и спектр., 78:2 (1995), 300.

[26] А. Снайдер, Дж. Лав, Теория оптических волноводов, Радио и связь, М., 1987.

[27] Н. Д. Кундикова, В. Г. Николаев, Н. Р. Садыков, М. О. Садыкова, Onm. и спектр., 96 (2004), 338.

[28] Б. А. Дубровин, С. П. Новиков, А. Т. Фоменко, Современная геометрия. Методъ и приложения, Наука, М., 1986.

[29] Н. Р. Садыков, Квантовая электроника, 22 (1995), 628.

[30] С. М. Рытов, ДАН СССР, 18 (1938), 263.

[31] Н. Д. Кундикова, В. Г. Николаев, Н. Р. Садыков, М. О. Садыкова, Onm. и спектр., 94 (2003), 699.

[32] Н.Р. Садыков, Квантовая электроника, 24 (1997), 190; Опт. атмосф. и океана, 11:3 (1998), 223; Onm. u cпектр., 86 (1999), 307; 90 (2001), 812.

[33] Д. Андерсон, Дж. Таннехил, Р. Плетчер, Вычислительная гидромеханика и теплообмен, Мир, М., 1990.

[34] Н. Р. Садыков, ТМФ, 135:2 (2003), 280; 139:3 (2004), 491; $\mathbf{1 4 4 : 3 ~ ( 2 0 0 5 ) , ~} 555$.

Поступила в редакцию 10.11.2005, после доработки 9.02.2006 